DOI: $10.37943 /$ AITU.2020.22.12.008

UDC: 65.012 .32

\author{
S. Bushuyev \\ Dr. Sc., Professor, Head of Project Management Department \\ sbushuyev@ukr.net,orcid.org/0000-0002-7815-8129 \\ Kiev National University of Construction and Architecture, Ukraine \\ D. Bushuyev \\ PhD, Associate Professor, Department of Information Technology \\ bushuyevD@gmail.com, orcid.org/0000-0001-5340-5165 \\ Kiev National University of Construction and Architecture, Ukraine
}

\title{
S. Neizvestny
}

Doctor of Technical Sciences, Professor, Department of Business Informatics sergey@neizvestny.com,orcid.org/0000-0002-5047-8251

Financial University under the Government of the Russian Federation, Russia

\section{CONVERGENCE AND HYBRIDIZATION OF PROJECT MANAGEMENT METHODOLOGIES}

\begin{abstract}
The subject of this article is the development of hybrid methodologies for managing projects, programs and project portfolios. Significant reductions in the life cycles of infrastructure programs, which contain projects of various lines of activity and various management methodologies, for example, waterfalls with a rigid life cycle and Agile with a flexible methodology life cycle, require the use of convergence tools to form "hybrid" methodologies. The goal is to develop a convergent approach to building hybrid project management methodologies in terms of decision-making processes in project management based on various platforms. The results of the study were obtained based on of using a convergent approach to build a methodology for managing infrastructure projects and programs. A substantial model of the obtained hybrid methodology for project and program management is presented. Conclusions: Hybrid methodologies for project and program management are becoming more popular in this category of programs. The main reason is the presence in the program of components with different life cycles, and, consequently, methodologies that require integration and harmonization. The methodology is tested by the practice of using the mechanisms of hybrid multi-level systems for managing infrastructure projects and programs. A study of hybrid project and program management methodologies defined by the practice of introducing various, in fact, program components. The use of a hybrid project management methodology allowed the authors to carry out various, in fact, projects on time with a specified budget. On the example of complex construction projects and projects for the creation of information and communication infrastructure management systems. Further areas of research are associated with a detailed study of the mechanisms of harmonization, integration, convergence and updating.
\end{abstract}

Keywords: model convergence, hybrid management methodologies, program, interaction model of basic project management methodologies, program manager. 


\title{
Бушуев С. Д.
}

Доктор технических наук, профессор, заведующий кафедрой управления проектами sbushuyev@ukr.net,orcid.org/0000-0002-7815-8129

Киевский национальный университет строительства и архитектуры, Украина

\section{Бушуев Д. А.}

Кандидат технических наук, доцент кафедры информационных технологий, bushuyevd@gmail.com,orcid.org/0000-0001-7298-4369

Киевский национальный университет строительства и архитектуры, Украина

\section{Неизвестный С. И.}

Доктор технических наук, профессор, кафедры «Бизнес-информатика»

sergey@neizvestny.com, orcid.org/0000-0002-5047-8251

Финансовый университет при Правительстве Российской Федерации, Россия

\section{КОНВЕРГЕНЦИЯ И ГИБРИДИЗАЦИЯ МЕТОДОЛОГИЙ УПРАВЛЕНИЯ ПРОЕКТАМИ}

\begin{abstract}
Аннотация: Предметом статьи является разработка гибридных методологий управления проектами, программами и портфелями проектов. Существенные сокращения жизненных циклов инфраструктурных программ, которые содержат проекты различных направлений деятельности и различными методологиями управления, например «водопадные» с жестким жизненным циклом и Agile с гибким жизненным циклом методологии, требуют использования инструментов конвергенции для формирования «гибридных» методологий. Цель - разработать конвергентный подход к построению гибридных методологий управления проектами с точки зрения процессов принятия решений в управлении проектами на основе различных платформ. Результаты исследования получены на основе использования конвергентного подхода к построению методологии управления инфраструктурными проектами и программами. Представлена содержательная модель полученной гибридной методологии управления проектами и программами. Выводы: гибридные методологии управления проектами и программами становятся более востребованными в этой категории программ. Главной причиной является наличие в программе компонентов с различными жизненными циклами, а следовательно, и методологиями, которые требуют интеграции и гармонизации. Методологию проверено практикой использования механизмов систем гибридного многоуровневого управления инфраструктурными проектами и программами. Исследование гибридных методологий управления проектами и программами, определенных практикой внедрения различных, по сути, компонентов программ. Применение гибридной методологии управления проектами позволило авторам выполнить различные, по сути, проекты в оговоренные сроки с заданным бюджетом. На примере сложных строительных проектов и проектов создания информационно-коммуникационных систем управления инфраструктурой. Дальнейшие направления исследований связаны с детальной проработкой механизмов гармонизации, интеграции, конвергенции и актуализации.

Ключевые слова: конвергенция моделей, гибридные методологии управления, программа, модель взаимодействия базовых методологий управления проектами, менеджер программы.
\end{abstract}

\section{Введение}

В практическом бизнесе предприятия часто сталкиваются с проблемой подбора оптимальных методологий, необходимых для эффективного управления портфелем программ и проектов. Как правило, в конкретных уникальных условиях реализации программ и про- 
ектов методологам предприятия сложно выбрать «подходящую» к целям и сфере функционального охвата проектов методологию из представленных на мировом или национальном «рынке» методологий [1-3]. Если же системно подойти к проблеме выбора методологии, то очевидно, что «коробочный» продукт в подобной ситуации не может идеально подойти, поскольку этот продукт разрабатывался для определенных условий применения, которые не могут совпадать с условиями на этом предприятии. Данная проблема ставит перед методологами предприятия задачу либо адаптировать «коробочную» методологию, либо разработать свою, под данный портфель, под условия, в которых работает предприятие. Оптимальная методология управления проектами будет направлять руководителя проекта через контролируемый, управляемый и видимый набор мероприятий для достижения результатов проекта $[4,5]$.

Однако разработка новой качественной методологии весьма затратное мероприятие, требующее высококвалифицированных методологических ресурсов, разностороннего профессионального опыта, времени и т.д. В профессиональных случаях, при решении данной проблемы, менеджеры-методологи ${ }^{1}$ идут по пути адаптации имеющихся методологий. Адаптация методологий может идти с применением конвергенции и или гибридизации.

Под конвергенцией методологий управления проектами (УП) будем понимать - системно выверенное слияние, объединение методологий при соблюдении условий непротиворечивости элементов объединяемых методологий. Конвергенция методологий предполагает эволюционное сближение/трансформацию некоторых элементов разных методологий в новые или модифицированные элементы. Это сближение происходит в основном под действием внешних и внутренних условий проектной деятельности. Основная цель применения механизмов конвергенции методологий - синергия элементов конвергируемых методологий при разработке методологии управления проектами для конкретных условий [6].

Под гибридизацией методологий будем понимать объединение элементов нескольких разных методологий в одну. Эту методологию можно назвать гибридной. В гибридной методологии элементы исходных методологий не подвергаются трансформаций.

Онтологически конвергенция методологий УП возникает под воздействием внешних и внутренних условий в ситуации достаточно масштабных, многофункциональных проектов и/или программ [7]. Выполняя сложные, многофункциональные программы проектов, руководство предприятия осознанно или нет логически вынуждено следовать закону Эшби: для эффективного управления разнообразием проектов, входящих в программу, необходимо иметь набор методологий больший, чем разнообразие проектов. Это разнообразие определяется несколькими факторами, например, целесообразностью экономии (не распыления) ресурсов: функционально специфический проект, с точки зрения экономической целесообразности, требований к качеству, времени, других параметров реализации проекта, диктует выбор специфической методологии, оптимальной для данного типа проектов. Если на предприятии методологи системно подходят к применению разных методологий, «специализированных» для конкретных проектов предприятия, то эволюционно начиная с масштаба жизненного цикла портфеля проектов, внутри жизненного цикла предприятия, под действием как внутренних, так и внешних условий реализации проектов, у применяемых разных методологий появляются некоторые общие характеристики [1,7-8]. Со временем у набора методологий появляются признаки «сходимости»³, на предприятии появляется единая «новая» методология, эффективно работающая в рамках этого предприятия. Это является прямым результатом конвергенции методологий.

\footnotetext{
${ }^{1}$ Следует заметить, что менеджеры большинства предприятий вообще не задумываются над данной проблемой: берут либо первую попавшуюся методологию «попроще», либо методологию, которой пользуются все «нормальные профессионалы».

2 Закон необходимого разнообразия.

${ }^{3}$ Разнообразие в предельном случае «вырождается» в одну-две методологии.
} 
Конвергенция методологий УП является объективным следствием воздействия условий реализации проектов. Это возможно именно в условиях высокого уровня зрелости процессов управления - не ниже третьего уровня по системе IPMA-Delta. Другими словами, в условиях высокой зрелости УП конвергенция неизбежно протекает, без решающей активной роли методологов, а под воздействием, прежде всего корпоративной культуры и системности процессов управления [7].

В условиях низкого уровня зрелости УП конвергентные проявления в развитии методологий незначительные и могут протекать в небольшом масштабе или вообще отсутствовать.

По-другому обстоит дело с использованием гибридизации методологий. В условиях невысокой зрелости УП, при наличии в организации профессиональных методологов, гибридизация методов УП может быть весьма эффективной. Опытный методолог УП может взять две или больше методологий УП, которые напрямую не совсем подходят для реализации проектов портфеля предприятия, создать оптимальный гибридный вариант единой методологии, отобрав у каждой из изначальных методологий те элементы, которые необходимы в специфике конкретного портфеля проектов данного предприятия.

В этом процессе создания гибридной методологии разработчик, используя принцип Оккама 4 , отсекает «ненужные» элементы (функционально не востребованные или элементы, дублирующие функции) методологий. Таким образом, гибридизация методологий является результатом активного воздействия методологов на изначальный «входной» набор методологий.

В условиях высокого уровня зрелости УП может использоваться и конвергенция, и гибридизация методологий. В этом комбинированном процессе конвергенция фактически может протекать как синергетическое объективное действие трансформации методологий при относительном незначительном («пассивном») воздействии методологов, а гибридизация - наоборот реализуется при активном воздействии методолога, действующего подобно скульптору, отсекающего от камня-заготовки все «лишнее».

В целом, с точки зрения параметров времени и изменения структур управления в развитии методологий УП, технологии конвергенции можно условно отнести к эволюционным, а технологии гибридизации - к революционным. При этом следует отметить, что конвергенция и гибридизация могут осуществляться одновременно, давая синергетический эффект.

Конвергенция и гибридизация подразумевают создание методологии для сложных многофункциональных проектов состоящей не только из сравнительно небольшого набора базовых элементов, но из расширенного набора, отвечающего всему разнообразию характеристик проекта, критериям его успешности, особенностям организационной структуры, в которой он реализуется [8-9]. т. е., обе эти технологии эффективны для «тяжелых» многоэлементных методологий [3].

Конвергентные технологии развития методологий УП сравнительно хорошо разработаны (см. например [6,7]) здесь мы их затрагиваем лишь частично, остановимся чуть подробнее на некоторых аспектах технологий гибридизации методологий УП.

\section{Гибридизация методов УП в условиях цифровизации и среды искусственного интеллекта}

Наиболее плодотворно применение гибридизации методологий УП в жизни цифрового общества, в автоматических системах, в частности в системах киберменеджмента.

Практически все существующие методологий управления проектами разработаны для человеческой среды, обычного человеческого интеллекта. Однако искусственный интеллект пока еще значительно отличается от интеллекта человека. Основные отличия искусственного интеллекта от естественного заключаются, прежде всего, в эмпатических, чув-

\footnotetext{
${ }^{4}$ Смысл принципа - «не плоди сущности», в нашем контексте означает устранение ненужных, балластных элементов методологии.
} 
ственных способностях, использовании комплекса ощущений, в анализе невербальных потоков информации, в использовании коллективного бессознательного и наследственного (генетически передаваемого) информационного пространства. В некоторых методах УП важная роль отводится, например таким инструментам принятия управленческих решений как интуиция [10] и измененные состояния сознания [11]. Естественно, мы не вправе переносить традиционные методологии УП в среду киберменеджмента, инсталлировать их «один в один» в искусственный интеллект.

В современном цифровом обществе назрела большая потребность в разработке новых методологий УП и на нынешнем этапе этих разработок наиболее эффективным может быть гибридизация. После создания пула методологий УП для цифровой среды методами гибридизации, имеет смысл использовать и механизмы конвергенции.
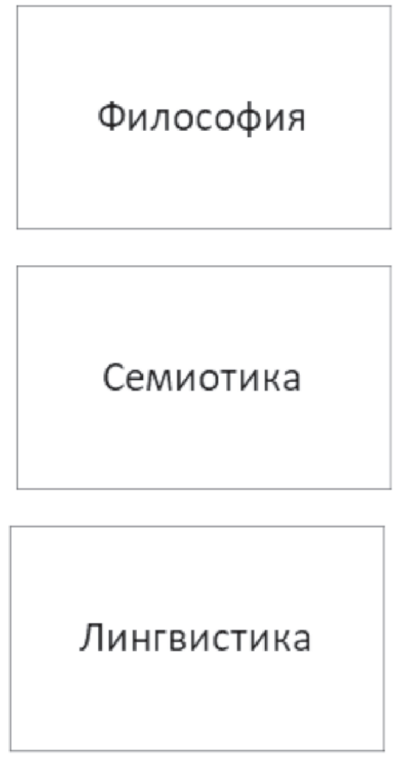
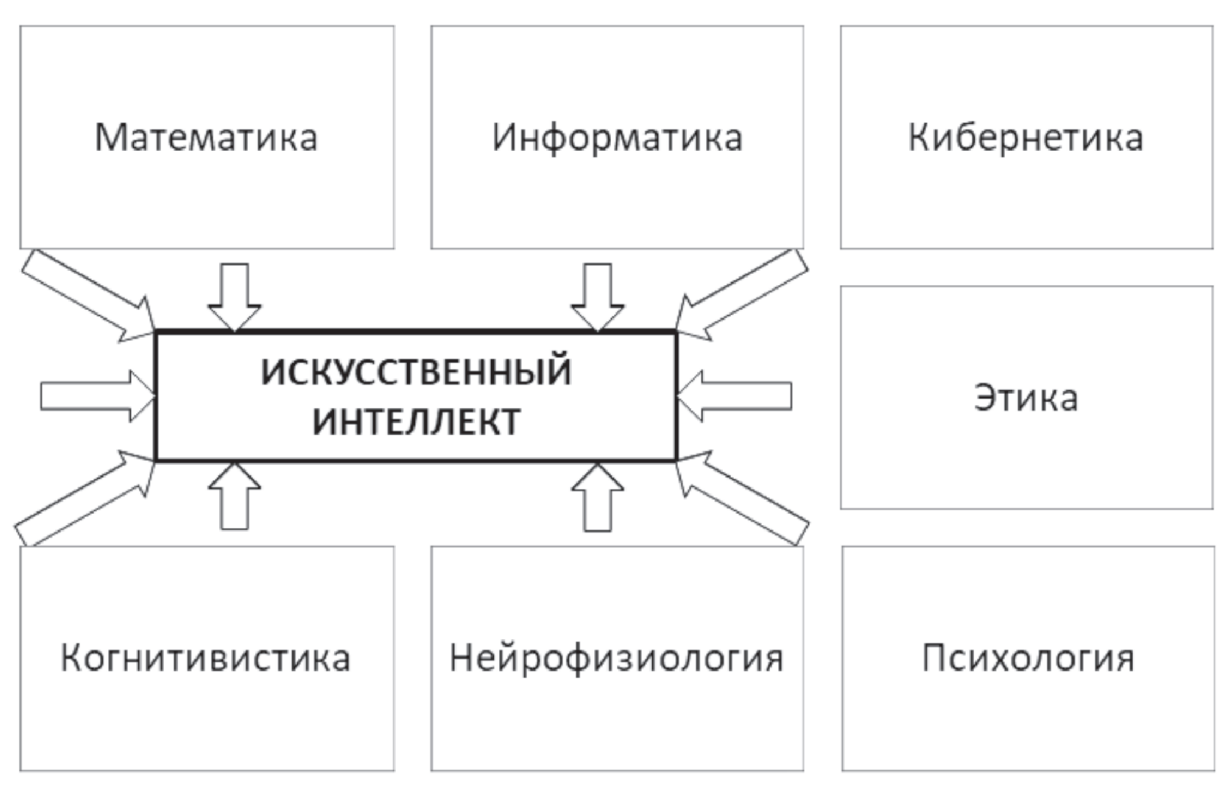

Рис. 1. Пример структуры областей знаний субъекта управления ИИ

Очевидно, что назрела потребность и в разработке интеллектуальных методологий УП, генерирующих методы управления в средах естественного, искусственного и комбинированного интеллектов.

Отличием при гибридизации разработки методологий УП является то, что в этих методологиях принципиально отличаются и объекты, и субъекты, и процессы управления [12]. Для этого достаточно взглянуть на пример структуры областей знаний субъекта управления ИИ (Рис. 1) и структуры гибридного процесса управления (Рис. 2). 


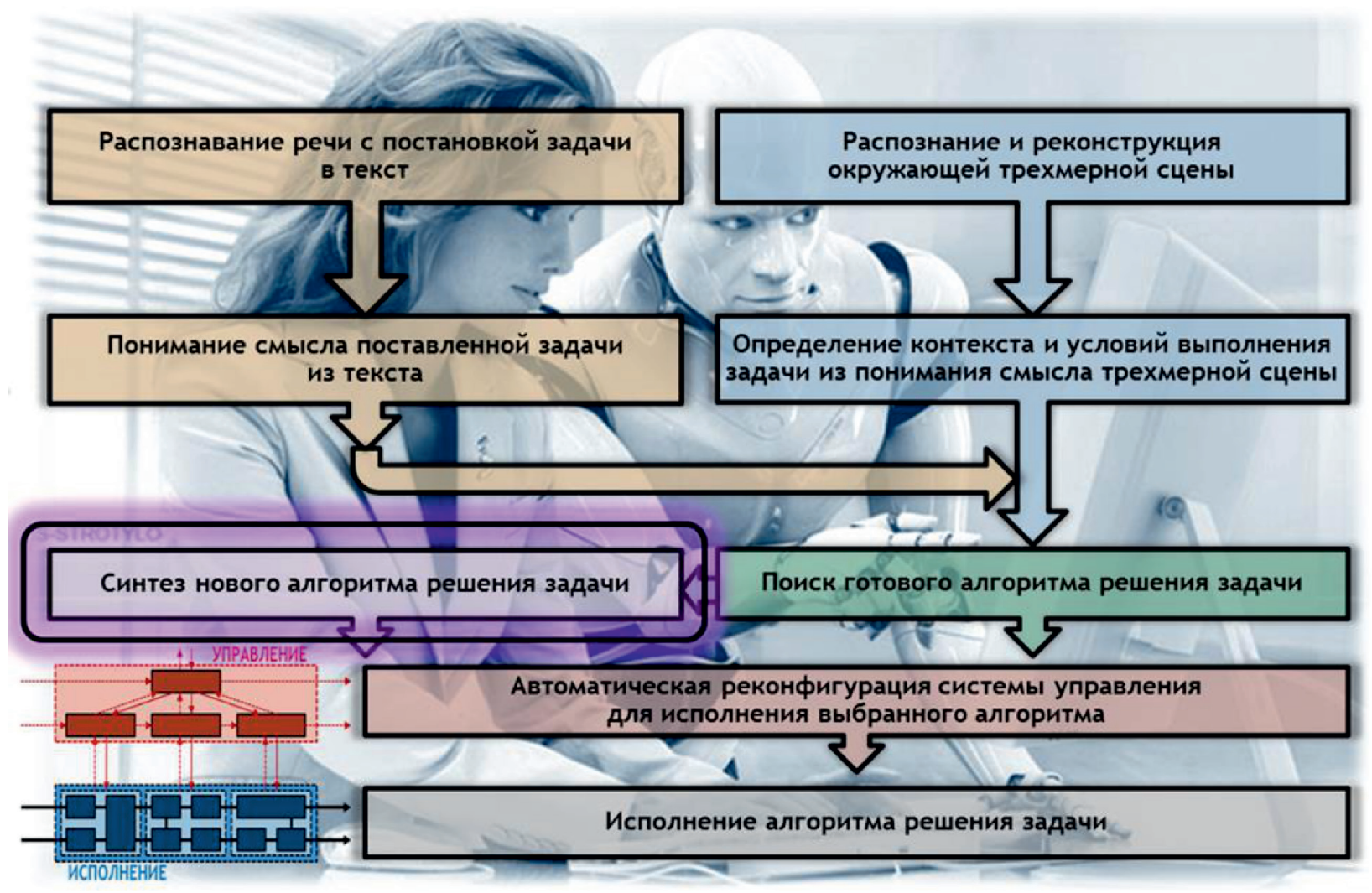

Рис.2. Структура части гибридного процесса управления «Естественный-искусственный» интеллект

Если в традиционных методологиях УП над многими аспектами, входящими, например в блок «Субъекты управления и поведенческая компетентность», влияющими на принятие решений мы не задумываемся, то в гибридных методологиях «Естественный-искусственный интеллект» эти аспекты требуют тщательной разработки. Гибридные методологии «Естественный-искусственный интеллект» нуждаются в расширении элементов, являющихся структурой функциональных поведенческих свойств ИИ (см. пример на Рисунке 3).

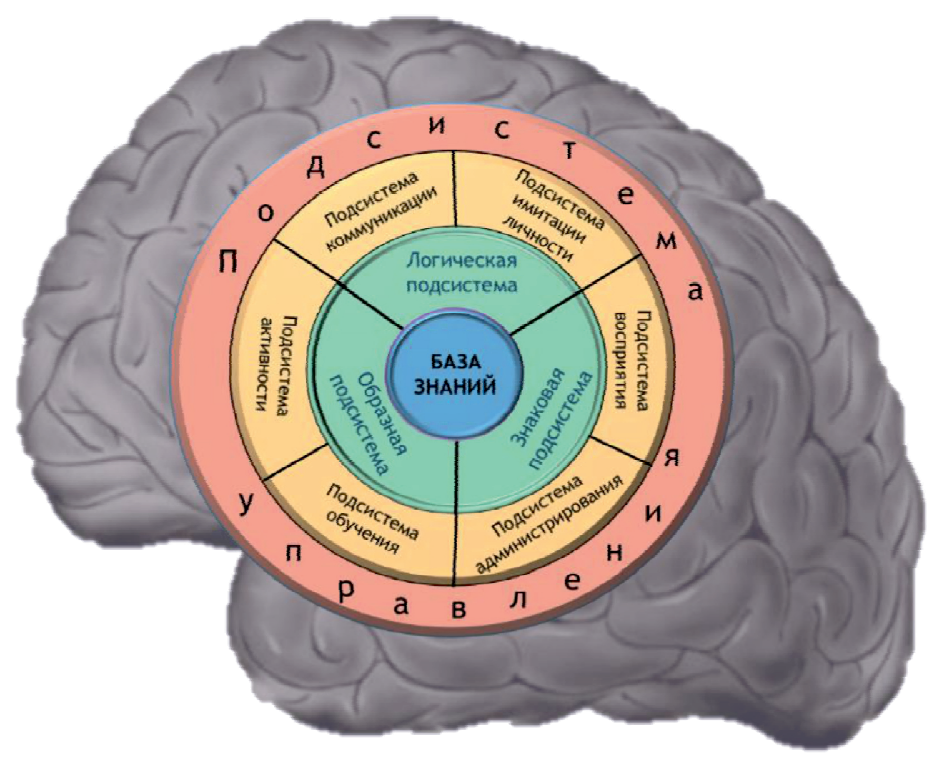

Рис. 3. Пример функциональной архитектуры ядра системы управления ИИ 
Гибридизация дает особый толчок развития систем поддержки принятия решений (СППР). Практическое применение современных СППР показывает их недостаточную эффективность, которая определяется как ограниченным количеством анализируемых связей «сущность-атрибут», так и ограничение учета влияния внешних и внутренних условий на процессы управления. Это не дает возможность отдать окончательное принятие решений искусственному интеллекту. Проблемы использования современных СППР возникают в социальном, и в юридическом аспектах. Так в социальном аспекте резко обостряется проблема утилизации высвобождающихся людских ресурсов, в юридическом аспекте возникают коллизии, связанные с так называемой проблемой «гильотины» для киберменеджера - ответственности за негативные последствия решений, принятых искусственным интеллектом $[13,14]$.

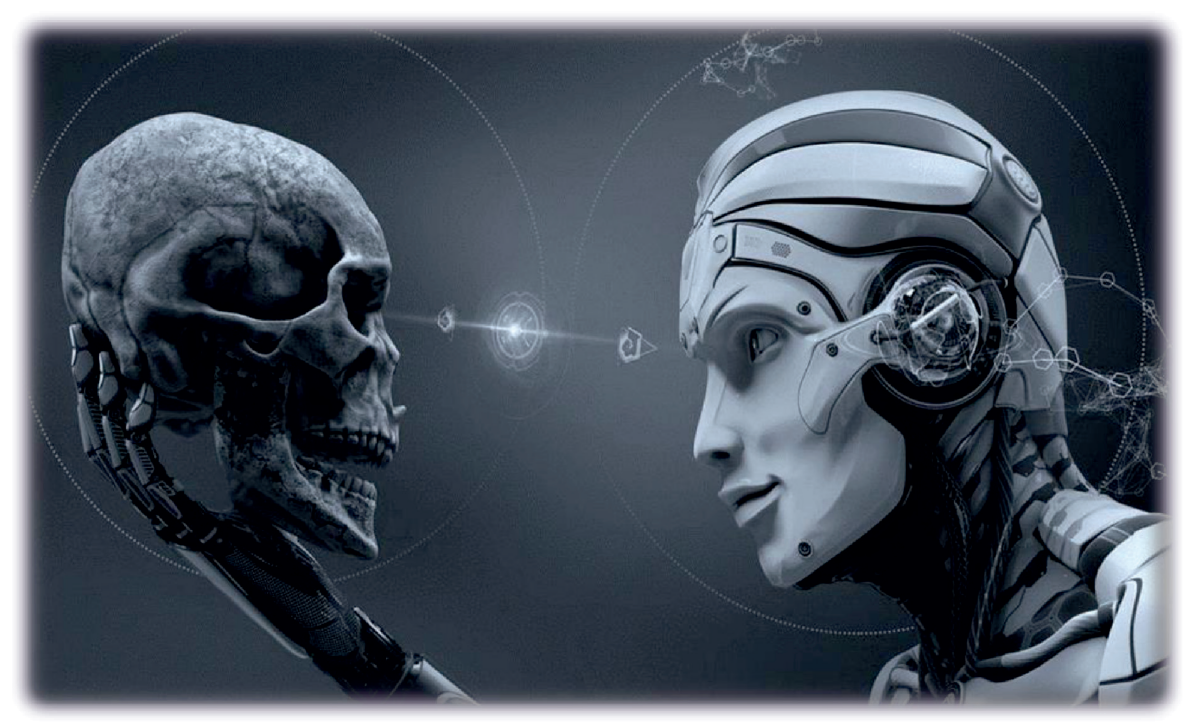

Рис.4. Для методологий УП искусственного интеллекта традиционные методологии могут представлять лишь ретроспективный интерес.

В будущем возможна ситуация, когда в гибридных методологиях УП искусственный интеллект полностью вытеснит современные (традиционные) методологии.

Гибридизация традиционных и кибернетических (на основе ИИ) методологий УП позволяют создать высокоэффективный «конвейер» решений, реальный экономический эффект способна принести лишь в случае полной трансформации бизнеса по принципу «АІ как процесс».

\section{Конвергенция, гибридизация и гибкие методологии УП}

Вопрос не в том, можно ли совмещать «традиционные» методологии УП и «новые, гибкие» методологии. Здесь проблема скорее заключается в терминологии и в замене простых сущностей. В современном бизнесе широко распространено мнение именно такое, что есть «старые» и «новые» методологии управления проектами. Если же рассмотреть фактическую сущность применяемых «старых» методологий, то она заключается в использовании хорошо спланированных, разработанных пакетов документов на весь жизненный цикл проекта. Начиная от концепции, технического, задания, паспорта, устава проекта и до интегрированного плана управления проектами со всеми регламентами и системно разработанными процессами управления (например, в соответствии с РМВОК РMI ICB IPMA). Этот вариант управления проектами применяется на тех предприятиях, где есть профессиональный пул менеджеров, методологов, специалистов в управления проектами и где 
нет «спешки» в реализации проектов, благодаря качественно проработанной стратегии бизнеса и качественному планированию. В таких организациях менеджеры, как правило, управляют ситуацией. В тех же случаях, когда уровень зрелости управления бизнесом не высок, там, где не достаточен профессионализм людей управляющих проектами, когда есть проблемы с планированием ресурсов, времени и т. д., когда возникает спешка, аврал, менеджеры переходят к так называемому «ручному» управлению. В условиях нехватки времени, как правило, возникшей из-за отсутствия профессионального долгосрочного планирования бизнеса (когда нет качественно проработанной стратегии планирования, реализации проекта, нет четкого единого понимания участниками цели, задач) команда проекта работает фактически без детального качественного технического задания, менеджеры начинают применять технологии (методологии) «гибкого» управления. По сути, гибкие методологии управления применяются в тех случаях, когда не менеджер управляет ситуацией, а наоборот, когда цели и задачи проекта корректируются, меняются по ходу его выполнения, когда методологические инструменты, средства меняются на ходу.

Рассмотрим концептуальную схему управления проектами и программами на основе гибридной методологии. Схема приведена на (Рис. 5)

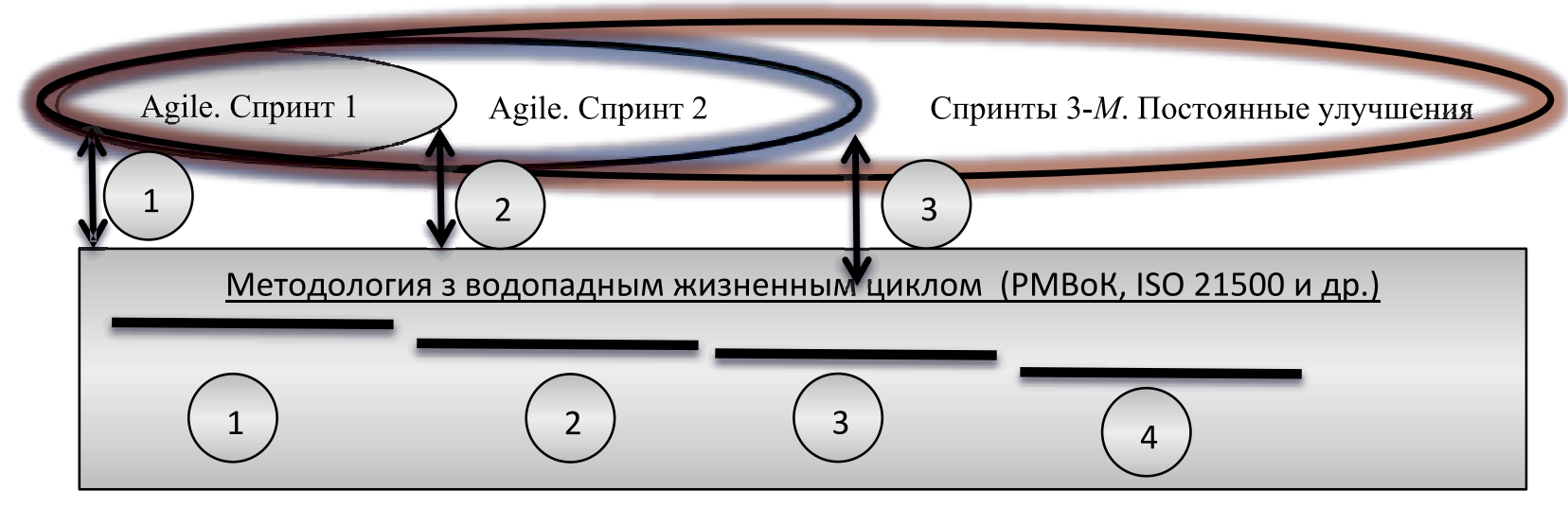

Рис. 5. Концептуальная схема управления проектами и программами на основе гибридной методологии

На данном примере приведена схема взаимодействия методологий Agile и PMBoK. B методологии Agile выделены два спринты. Первый спринт, где создается минимальный работающий продукт. Второй спринт, где создается работающий продукт с необходимыми функциями, поддерживающих функций этого компонента инфраструктурного проекта. Третий и последующие спринты, посвященные системе постоянных улучшений (Кайдзен). В методологии на основе водопадных моделей жизненного цикла, приведены четыре фазы проектов. На основе связей 1-3 модели гибридной методологии гармонизируются, интегрируются, проводится их определенная конвергенция и актуализация. Эти операции приведены на Рисунке 5 при формировании гибридной методологии управления инфраструктурными программами на основе геномных представлений компонентов.

Управлять успехом проектов и программ можно только ориентируясь на векторы создания ценностей для различных групп заинтересованных сторон, использовать тренды ускорения развития, конвергенции знаний, своевременной смены парадигм управления, понимания философии жизненных циклов. Каждый из этих элементов является составляющей успеха программ, а формула успеха определяется через синергию их взаимодействия [5,6].

Возврат от проактивного к реактивному методу управления, связан с трансформацией вызова в проблему. Сроком про активности назовем период трансформации вызова в проблему. Подход к оценке срока проактивности используют в проектах разработки 
программных систем на основе методологии Agile. Например, в таком определении, как проактивная защита проекта или программы означает совокупность технологий, которые используются в информационно-коммуникационных системах. Главной целью является выявление потенциально опасного программного обеспечения и устранения его влияния или самого программного кода в пределах работающих программных систем. В отличии от других технологий они предупреждают и пытаются блокировать опасную активность, а не обнаруживают уже известную злонамеренную программу в системе. При этом используется модель активных точек бифуркации, которые привязаны к событиям жизненного цикла и меняют приоритеты компонентов проекта или программы к внедрению одной из стратегий - антикризисной стабилизации и устойчивого развития.

Философия управления стейкхолдерами исходит из того, что их претензии являются легитимными и потому подлежат учету. При этом следует заметить, что те интерпретации концепции заинтересованных сторон, лимитируют легитимность их претензий, должны быть отклонены по экономическим соображениям, так как они игнорируют факт ограниченности ресурсов организации. Неограниченное удовлетворение требований невозможно, поскольку это вызовет дефицит обменного продукта. Со своей стороны заинтересованные стороны во многих случаях не намерены без сопротивления отказываться от своих претензий. На практике это приводит к возникновению политических процессов в отношениях между группами, в частности к борьбе за распределение материальных и других благ.

Рассмотрим обобщенную модель инфраструктурных программ в реализации, которых задействованы компаний ряда стран.

Модель представляется в виде.

$$
K=<\check{E}, \breve{O}>\text {. }
$$

Здесь $\check{E}$ - окружение инфраструктурной программы;

$\breve{O}$ - модель деятельности по внедрению инфраструктурной программы.

Окружение организации Ӗ рассматривается, как фрактал, состоящий из самоподобных моделей культурного, политического и экономического окружения стран, в которых ведется деятельность организации, которые задействованы во внедрении проектов и программ.

$$
\check{E}=\left\{E_{1}, E_{2}, E_{i} \ldots, E_{n}\right\}
$$

где $n$ количество стран, в которых осуществляет бизнес организации.

Согласно проектно-ориентированная организация содержит ряд обособленных подразделений в этих странах и отображается моделью фрактала Ď в организации самоподобные по структуре и функциям организаций.

$$
\check{D}=\left\{D_{1}, D_{2}, D_{j} \ldots, D_{m}\right\}
$$

где $m$ количество подразделений организации.

На основе подразделений $\check{D}$ организации формируется модель ее деятельности $\breve{O}$ на основе концептуальной схемы создания и миграции ценностей в культурном, политическом и экономическом среде. При этом подразделения организации в разных странах имеют самоподобные структуры. Это дает возможность применить фрактальные модели для формирования механизмов управления.

Модель окружения организации:

$$
\forall E_{i} \in \check{E} \exists M_{i}^{o}=<\check{\mathrm{R}}_{i}, \hat{\mathrm{G}}_{i}, \mathrm{C}_{i}>,
$$

где $M_{i}^{o}$ - модель окружения организации в $i$-й стране;

$\check{R} i$ - модель влияния рынка $i$ страны на деятельность организации; 
$\hat{G} i$ - модель взаимодействия с партнерами в данной стране;

$C i$ - модель взаимодействия с конкурентами в $i$-й стране.

Пусть модель деятельности в каждой стране формируется в виде

$$
\forall D_{j} \in \check{D} \exists O_{j}^{o}=<\mathrm{P}_{j}, \hat{\mathrm{R}}_{j}, \hat{\mathrm{U}}_{j}>,
$$

где $M_{i}^{o}$ - модель деятельности организации в ј-й стране;

$P j$ - модель создания продукции в ј-й стране организации;

$\hat{R} j$ - модель производства в ј-й стране;

$\hat{U j}$ - модель управления в ј-й стране.

Модель взаимного влияния определяется матрицами $\Psi p, \Psi n$, $\Psi$ кыраженными в форме мультипликаторов, которые взаимодействуют через демпферы или приемники рисков влияния рынка на организацию, партнеров и конкурентов. Здесь $\Psi р$ - матрица влияния окружения на организации; $\Psi n$ - матрица влияния окружения на партнеров; $\Psi к-$ матрица влияния окружения на конкурентов. Элементы матриц $\Psi p[1, j], \Psi n[1, j], \Psi \kappa[1, j]$ определяют влияние окружения на деятельность подразделения ј организации. Элементы матриц $\Psi p[2, j], \Psi n[2, j], \Psi \kappa[2, j]$ определяют влияние деятельности подразделения ј организации на окружение. Модель влияния, которая определяет иммунные механизмы, включая коэффициенты, распределены по пяти зонам риска воздействий критических факторов на состояние организации и будущие разрушения. Модель основана на трендах, мониторинг которых осуществляется командой проекта по шкале оценок $\Omega$ :

Пусть для каждого элемента матриц $\Psi p[1, j], \Psi n[1, j], \Psi \kappa[1, j]$ известны $F p(\Omega j), F n$ $(\Omega j), F \kappa(\Omega j)$ механизмы управления гибридной методологии - демпферы угроз от рынка и конкурентов и поглотители рисков, переданных партнерам инфраструктурной программы.

Тогда механизм управления инфраструктурными программами организации реализует для каждого подразделения такие шаги:

1. Оценка состояний окружения для каждого подразделения организации $\Omega j$.

2. Для каждого подразделения организации оцениваются значения поглощения рисков из-за их передачи партнерам по контрактам и компании в целом.

$$
\forall j=\overline{1, J}: F_{\Pi}^{\mathrm{MK}}=\sum F_{\Pi}(\Omega j),
$$

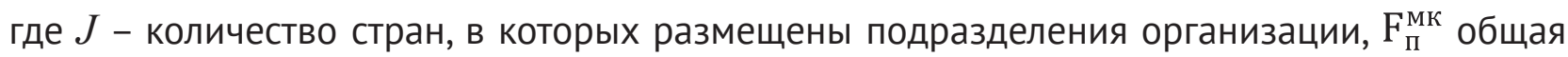
сумма потенциальных потерь, переданных партнерам.

3. Для каждого подразделения организации оцениваются значение принятых угроз, рисков поведения рынка и возможного демпфирования их негативного влияния на организации целом.

$$
\forall j=\overline{1, J}: F_{\mathrm{p}}^{\mathrm{MK}}=\sum F \mathrm{p}(\Omega j)
$$

где $F_{\mathrm{K}}^{\mathrm{M \kappa}}$ - общая сумма потенциальных потерь от негативного влияния рынка в ј-й стране.

4. Для организации оцениваются значение принятых угроз, рисков и частичной их передачи пантерам

$$
\Phi=F_{\mathrm{p}}^{\mathrm{MK}}+F_{\mathrm{K}}^{\mathrm{MK}}-F_{\Pi}^{\mathrm{MK}}
$$

где $\Phi$ - общий объем потерь от негативного влияния окружения инфраструктурного программы.

5. Выделяется значение потерь, которые формируют максимальные отрицательные влияния рынка или конкурентов. 


$$
\forall j=\overline{1, J}, \Psi \mathrm{e}=\max \left(F p(\Omega j), F_{\mathrm{K}}(\Omega j)\right)
$$

6. Для каждого максимального значения потерь Же формируется антикризисный проект, в котором оцениваются затраты. Это и индикатор $B C R$ - выгоды к затратам. Если коэффициент $B C R$ больше 1, то этот проект размещается в пул проектов антикризисного управления.

7. Если существуют еще не рассмотреные $F p(\Omega j), F_{\kappa}(\Omega j)$, то происходит очередная итерация и осуществляется переход к п. 3.

8. После формирования пула проектов инфраструктурной программы происходит их отбор в текущий портфель проектов. При этом отбор проектов происходит с учетом ограничения

$$
\sum_{1}^{\mathrm{e}} \mathrm{Ce} \leq B p
$$

где $B p$ - бюджет инфраструктурной программы на текущий период времени.

С целью построения эффективной гибридной методологии управления инфраструктурными проектами обоснована целесообразность использования геномных представлений методологий управления портфелем, программами и проектами организации $[5,6]$.

Проведем анализ применения геномных представлений методологий управления с учетом существующих проблем финансовых организаций, турбулентности внутреннего и внешнего окружения. Проблемы распределим на четыре группы: определение цели, целедостижения, организационной компетентности и организационного поведения (Рис.6).

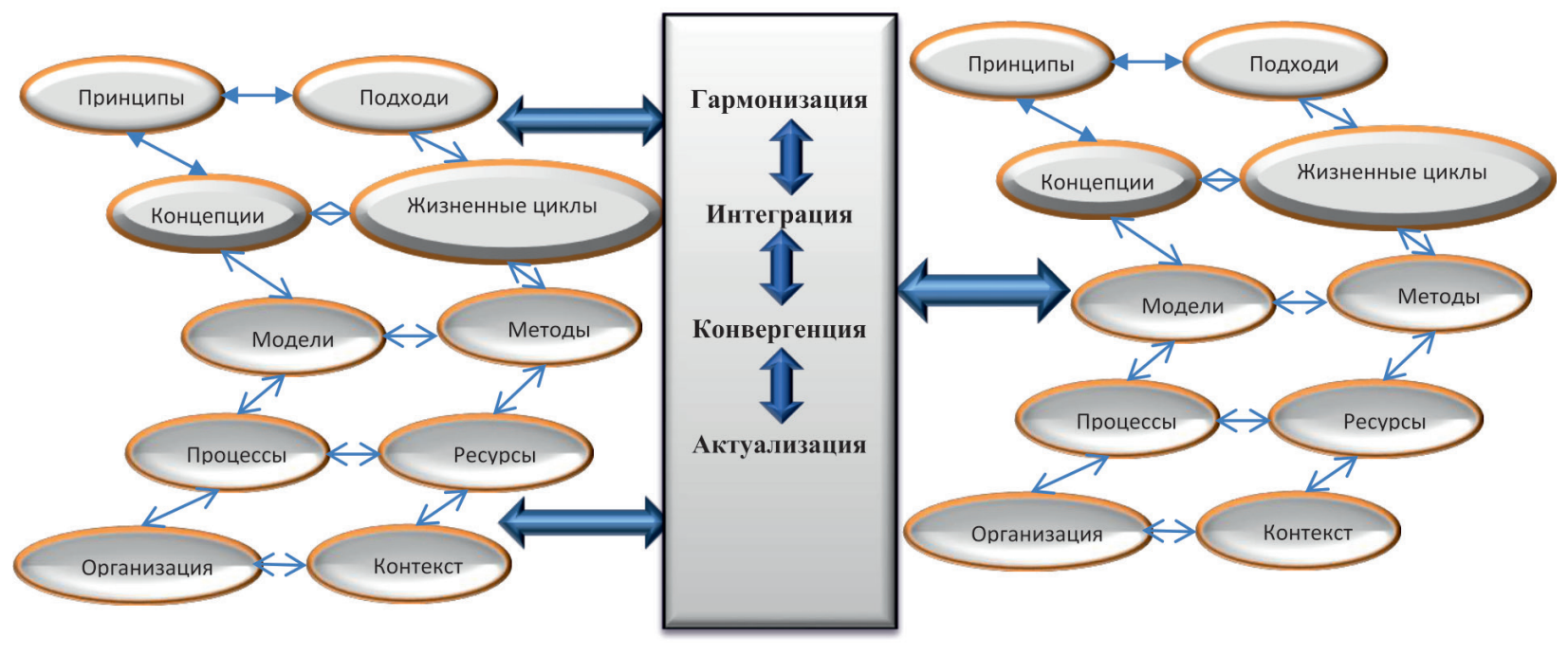

Методология 1

Методология 2

Рис. 6. Модель генома гибридной методологии управления проектами и программами

В качестве примера рассмотрим поэтапное формирование методологий. Применение механизмов взаимодействия зависит от уровня зрелости управления проектами в организации [10]. На низких уровнях зрелости методология нужна не в полном объеме и может быть представлена не всеми элементами. На высоких уровнях зрелости организаций, реализующих масштабные, комплексные программы и проекты, нужна методология, которая обладает всем набором элементов, содержащих все разнообразие необходимых элементов. То есть для самого сложного и гипотетически разнообразного портфеля программ и проектов методология приближается к «гипотетически полной системе». 
Носитель знаний методологии, сформированный по этой модели. Формализованная модель генома методологий записана следующим образом.

$$
M=<P, K, A, L, \Pi, D, V, O>\text {. }
$$

Пусть известно множество принципов, определяющих методологию:

$$
P=\left\{p_{1}, p_{2}, \ldots p_{n}\right\} .
$$

Данная множество должна обладать свойствами полноты и непротиворечивости. В рамках реализации принципов и альтернативных концепций известно множество подходов, применяемых в определении методологии:

$$
A=\left\{a_{1}, a_{2}, \ldots a_{m}\right\} .
$$

На основе этих принципов могут быть сформированы альтернативные концепции:

$$
K=\left\{k_{1}, k_{2}, \ldots k_{i}\right\} .
$$

Методология должна быть приемлемой для множества жизненных циклов проектов:

$$
L=\left\{l_{l}, l_{2}, \ldots l_{r}\right\} .
$$

На объединение моделей накладываются модели и методы управления проектами, программами и портфелями проектов. Данные модели, методы и механизмы осуществляются на основе процессных составляющих методологии:

$$
\Pi=\left\{n_{1}, n_{2}, \ldots n_{q}\right\} .
$$

Документы, сопровождающие систему процессов управления, определяются в виде множества:

$$
D=\left\{d_{1}, d_{2}, \ldots d_{z}\right\} .
$$

Каждая методология привязывается к корпоративной культуре управления (культура выражается через набор культурных ценностей):

$$
V=\left\{v_{1}, v_{2}, \ldots v_{c}\right\}
$$

Данная методология должна быть привязана к организационной среде контекста:

$$
O=\left\{o_{1}, o_{2}, \ldots o_{g}\right\} .
$$

Где индексы $n, m, s, r, q, z, c, g$ определяют количество элементов каждого множества, которое описывает геном методологий.

В данной модели выделим два механизма: систематизации в рамках каждого уровня и гармонизации между уровнями модели.

Механизм гармонизации обеспечивает системную обработку моделей каждого уровня. При этом элементы каждого уровня систематизируются на основе матрицы смежности.

Механизм интеграции обеспечивает формирование целостной модели гибридной методологии. Такая интеграция зависит от специфики каждой методологии, которая включается в гибрид.

Механизм конвергенции обеспечивает взаимопроникновение лучших практик (решений) в пределах применяемых методологий.

Механизм актуализации готовит гибридную методологию внедрения. Каждый из этих механизмов имеет сложную структуру, функции входа и выхода. Такие механизмы будут подробно определены в следующих публикациях авторов. 
Например, взаимосвязи на первом уровне между принципами и подходами систематизируются матрицей смежности. При этом элементы матрицы определяют системную совместимость отдельных принципов и подходов. Значение 0 определяет несоответствие элементов или отсутствие их взаимовлияния, 1 - полную согласованность и максимальное взаимовлияние. Аналогично формируются матрицы на других уровнях модели. Задача механизма систематизации - это определение системной совместимости выбранных элементов методологий. В данном случае под термином «генетический код проекта (программы)» будем понимать его системную модель, которая включает первоначальное представление о «видение» продукта, проекта или программы, интегрированный процесс развития определенной функциональной деятельности, построенный для всего жизненного цикла проекта, инструменты его взаимодействия с внешней средой. При этом геномы методологий управления проектами, управления портфелями проектов и программ имеют одинаковую спиральную структуру для разного наполнения. Введем понятие алгебры методологий управления проектами:

$$
A=<\check{S}, \Omega>,
$$

где $\check{S}$ - носитель знаний методологий, размещается в геноме;

$\Omega$ - сигнатура, что определяет множество операций над элементами методологий.

Сигнатура содержит операции проекции отдельных элементов методологии и их групп, объединения, пересечения и дополнения методологий и т. д.

Назначением генетического кода проектов, программ и портфелей является навигация в реализации функций и процессов развития. При этом навигатор, продвигаясь по фазам и шагам жизненного цикла, использует информацию генетического кода - его взаимосвязанные структуры в процессах управления. Чаще всего процесс формирования генетического кода проекта происходит стихийно, на основе интуиции и практики управления проектами организации. Двигаясь дальше в применении аналогий управления проектами в области геномных моделей, выделим два дополнительных генома гибридной модели геном компетенций по Методологии 1 и геном компетенций по Методологии 2. Эти два генома пересекаются в точке, где осуществляется реализация компетенций проекта за счет компетентности проектных менеджеров. Такое взаимодействие формирует «хромосому» проекта [6].

Можно ли говорить, что «гибкие» методологии используют гибридные технологии. Да можно, когда по ходу выполнения проекта одна методология (фрагмент методологии) заменяется или дополняется другой методологией или имеет место методологическая импровизация [5]. Однако во многих случаях применения «гибких» методологий, гибридизация напоминает не системно продуманные и заранее запланированные процессы синергетического совмещения определенных методологий, а спорадические действия в стихийно сложившемся «зоопарке» методологий. Соответственно в «гибких» методологиях УП конвергенция методологий может присутствовать лишь фрагментарно, а чаще вообще отсутствует.

Если рассмотреть в качестве примера «гибкой» методологии УП средство «Agile» и смежные с ним инструменты (КАНБАН, СКРАМ и др.), то ретроспективный анализ УП показывает, что принципиально нового с точки зрения прибовлении знаний в управлении здесь ничего нет. Так, например еще в 1924 году в Центральном институте труда под руководством А.К. Гастева были разработаны методологии бережливого производства, эргономики труда и гибких способах планирования и реализации работ в условиях нехватки времени и других ресурсов. Другие примеры по этому поводу можно найти в системных научных исследованиях и обзорах (см. например, [20]), которые в целом склонны к выводу в отношении «гибких» методологий [16-19]. 
«Новое - это новая комбинация старого в новых условиях». В современном бизнесе Agile активно применяется в экстремальных условиях, в частности, в проектах экстремального программирования. Однако с системной точки зрения создание экстремальной ситуации является следствием недостаточно зрелого процесса управления [21-25].

Несмотря на свою методологическую фрагментарность, но и то, что «гибким» методологиям присущи дополнительные материальные, человеческие, а иногда и финансовые затраты ввиду неустойчивости по причинам реакции на внутренние и внешние изменения (реакции на ситуации). Эти методологии частично применяются и на предприятиях высокой зрелости управления обычно, как гибридное дополнение к основным методологиям. Гибридные методологии, построенные на базе традиционных с использованием ИИ и элементами «гибких» методологий, позволят снизить риски распыления ресурсов, повысить производительность и качество принятия управленческих решений [7, 20].

\section{Заключение}

Применение технологий конвергенции и гибридизации для создания или адаптации методологии управления проектом определяется характеристиками проекта, его сложностью, масштабом, требованиям к критериям успешности, организационной структурой, в которой он реализуется. Однако эффективное использование этих технологий во многом зависит от уровня компетентности руководителя проекта, коллективной компетентности и в целом уровня зрелости управления проектами на предприятии [26].

С точки зрения формирования и развития индивидуальных и особенно коллективных баз знаний и компетенций в УП, гибридные методологий могут значительно увеличить системный охват мирового опыта, повысить скорость накопления знаний, производительность и качество принятия решений.

Не уместным является подход, когда в силу сложившейся ситуации на предприятии под принятую на нем методологию подгоняется управление проектами: в организациях зрелого бизнеса используется обратный подход - для эффективной реализации проектов под них выбираются, адаптируются или разрабатываются конкретные методологий. В последнем случае продуктивно применяются и конвергенция, и гибридизация методологий.

\section{Литература}

1. Collyer, S., Warren, C., Hemsley, B. \& Stevens, C. (2010) Aim, fire, aim - Project planning styles in dynamic environments. Project Management Journal, 41(4), 108-121.

2. Nelson, K.M., Ghods, M. \& Nelson, H.J. (1998). Measuring the effectiveness of a structured methodology: a comparative analysis. Proceedings of the Thirty-First Hawaii International Conference on System Sciences, Kohala Coast, HI, 492-499.

3. Cockburn, A. (2000). Selecting a Project's Methodology. IEEE Software, 17(4), 64-71.

4. Office of Government Commerce. (2009). Managing Successful Projects with PRINCE 2. Norwich, UK: The Stationary Office.

5. Бушуев С.Д., Бушуева Н.С., Бабаев И.А., Яковенко В.Б., Гриша Е.В., Дзюба С.В., Войтенко А.С. (2010). Креативные технологии управления проектами и программами: Монография. - К.: «Саммит-Книга», 768.

6. Бушуев С.Д., Бушуева Н.С., Неизвестный С.И. (2012). Механизмы конвергенции методологий управления проектами. Управление развитием сложных систем. Вып. 11. Киев, 5-13.

7. Неизвестный С.И. (2019). Конвергентные технологии развития методологий управления проектами. Москва - Санкт-Петербург: Нестор-История, 352.

8. Saynisch M. (2010). Beyond frontiers of traditional project management:An approach to evolutionary, self-organizational principles and the complexity theory - Results of the research program. Project Management Journal, 41(2), 21-37. 
9. Bushuyev S. \& Wagner R. (2014). IPMA Delta and IPMA Organizational Competence Baseline (OSB): New approaches in the field of project management maturity. International Journal of Management Projects in Business, 7(2), 302-310.

10. Бушуев С.Д., Неизвестный С.И. (2011). Шестое чувство в управлении развитием: интуиция как канал коммуникации и средство принятия решений. Управление развитием сложных систем. Киев, вып. 7, 8-15.

11. Неизвестный С.И., Черных Н.В. (2012). Роль измененных состояний сознания при принятии решений в менеджменте. Управление проектами и программами. №1, 56-65; №2, 114-123.

12. Luger G.F. (2008). Artificial Intelligence. Structures and Strategies for Complex Problem Solving. 6 Edition. University of New Mexico: Pearson, 784.

13. Бурков В.Н., Буркова И.В. (2018). Цифровая экономика и умные механизмы принятия решений. Управление проектами и программами, №2 (54), 118-125.

14. Клименко Э.Ю., Неизвестный С.И. (2018). Трансформация управления проектами в цифровой экономике. Управление проектами и программами. №2 (54), 110-117.

15. Leybourne, S.A. (2009). Improvisation and agile project management: A comparative consideration. International Journal of Managing Projects in Business, 2(4), 519-535.

16. Bailes K. E. (1977). Alexei Gastev and the Soviet Controversy over Taylorism 1918-1924 Soviet Studies. Glasgow, UK.

17. Гастев А.К. (2011). Как надо работать: Практическое введение в науку организации труда. Советский производственный менеджмент. Изд. 3. М.: УРСС, 480.

18. Гастев А.К. (2011). Трудовые установки. М.: УРСС, 344.

19. Журавский А.Ф. (1926). Научная организация труда. М.; Л.: Госиздат, 220.

20. Spundak M. (2014). Mixed agile/traditional project management methodology - reality or illusion? 27th IPMA World Congress. Procedia - Social and Behavioral Sciences, Vol.119, 939-948.

21. Маршев В.И. (2019). XX Международная конференция «История управленческой мысли и бизнеса. Менеджмент и роли менеджеров: вчера, сегодня, завтра». Москва, 28-30 июня 2019 г. Сборник трудов конференции. М.: МГУ, 288.

22. Conforto, E.C. \& Amaral, D.C. (2008). Evaluating an agile method for planning and controlling innovative projects. Project Management Journal, 33(4), 4-14.

23. Chow, T. \& Cao, D. (2008). A survey study of critical success factors in agile software projects. The Journal of Systems and Software, 81(6), 961-971.

24. Fernandez, D.J. \& Fernandez, J.D. (2008). Agile Project Management - Agilism versus traditional approaches. Journal of Computer Information System, 49(2), 10-17.

25. Boehm, B. \& Turner, R. (2005). Management challenges to implementing agile processes in traditional development organizations. IEEE Software, 22(5), 30-39.

26. Bushuyev S., Yashenko Yu., Tovb A., \& Neizvestny S. (2012, 29-31 October). System paradigm of collective and individual competences of project management specialists. Reports on 26th IPMA World Congress, Greece, Iraklion.

\section{References}

1. Collyer, S., Warren, C., Hemsley, B. \& Stevens, C. (2010) Aim, fire, aim - Project planning styles in dynamic environments. Project Management Journal, 41(4), 108-121.

2. Nelson, K.M., Ghods, M. \& Nelson, H.J. (1998). Measuring the effectiveness of a structured methodology: a comparative analysis. Proceedings of the Thirty-First Hawaii International Conference on System Sciences, Kohala Coast, HI, 492-499.

3. Cockburn, A. (2000). Selecting a Project's Methodology. IEEE Software, 17(4), 64-71.

4. Office of Government Commerce. (2009). Managing Successful Projects with PRINCE 2. Norwich, UK: The Stationary Office.

5. Bushuev S.D., Bushueva N.S., Babaev I.A., YAkovenko V.B., Grisha E. V., Dzyuba S.V., Vojtenko A.S. (2010). Kreativnye tekhnologii upravleniya proektami i programmami: Monografiya. - K.: «SammitKniga», 768.

6. Bushuev S.D., Bushueva N.S., Neizvestnyj S.I. (2012). Mekhanizmy konvergencii metodologij upravleniya proektami. Upravlenie razvitiem slozhnyh sistem. Vyp. 11. Kiev, 5-13. 
7. Neizvestnyj S.I. (2019). Konvergentnye tekhnologii razvitiya metodologij upravleniya proektami. Moskva - Sankt-Peterburg: Nestor-Istoriya, 352.

8. Saynisch M. (2010). Beyond frontiers of traditional project management:An approach to evolutionary, self-organizational principles and the complexity theory - Results of the research program. Project Management Journal, 41(2), 21-37.

9. Bushuyev S. \& Wagner R. (2014). IPMA Delta and IPMA Organizational Competence Baseline (OSB): New approaches in the field of project management maturity. International Journal of Management Projects in Business, 7(2), 302-310.

10. Bushuev S.D., Neizvestnyj S.I. (2011). SHestoe chuvstvo v upravlenii razvitiem: intuiciya kak kanal kommunikacii i sredstvo prinyatiya reshenij. Upravlenie razvitiem slozhnyh sistem. Kiev, vyp. 7, 8-15.

11. Neizvestnyj S.I., CHernyh N.V. (2012). Rol' izmenennyh sostoyanij soznaniya pri prinyatii reshenij v menedzhmente. Upravlenie proektami i programmami. № 1, 56-65; № 2, 114-123.

12. Luger G.F. (2008). Artificial Intelligence. Structures and Strategies for Complex Problem Solving. 6 Edition. University of New Mexico: Pearson, 784.

13. Burkov V.N., Burkova I.V. (2018). Cifrovaya ekonomika i umnye mekhanizmy prinyatiya reshenij. Upravlenie proektami i programmami, № 2 (54), 118-125.

14. Klimenko E.YU., Neizvestnyj S.I. (2018). Transformaciya upravleniya proektami v cifrovoj ekonomike. Upravlenie proektami i programmami. № 2 (54), 110-117.

15. Leybourne, S. A. (2009). Improvisation and agile project management: A comparative consideration. International Journal of Managing Projects in Business, 2(4), 519-535.

16. Bailes K.E. (1977). Alexei Gastev and the Soviet Controversy over Taylorism 1918-1924 Soviet Studies. Glasgow, UK.

17. Gastev A.K. (2011). Kak nado rabotat': Prakticheskoe vvedenie v nauku organizacii truda. Sovetskij proizvodstvennyj menedzhment. Izd. 3. M.: URSS, 480.

18. Gastev A.K. (2011). Trudovye ustanovki. M.: URSS, 344.

19. Zhuravskij A.F. (1926). Nauchnaya organizaciya truda. M.; L.: Gosizdat, 220.

20. Spundak M. (2014). Mixed agile/traditional project management methodology - reality or illusion? 27th IPMA World Congress. Procedia - Social and Behavioral Sciences, Vol.119, 939-948.

21. Marshev V.I. (2019). XX Mezhdunarodnaya konferenciya «Istoriya upravlencheskoj mysli i biznesa. Menedzhment i roli menedzherov: vchera, segodnya, zavtra». Moskva, 28-30 iyunya $2019 \mathrm{~g}$. Sbornik trudov konferencii. M.: MGU, 288.

22. Conforto, E.C. \& Amaral, D.C. (2008). Evaluating an agile method for planning and controlling innovative projects. Project Management Journal, 33(4), 4-14.

23. Chow, T. \& Cao, D. (2008). A survey study of critical success factors in agile software projects. The Journal of Systems and Software, 81(6), 961-971.

24. Fernandez, D.J. \& Fernandez, J.D. (2008). Agile Project Management - Agilism versus traditional approaches. Journal of Computer Information System, 49(2), 10-17.

25. Boehm, B. \& Turner, R. (2005). Management challenges to implementing agile processes in traditional development organizations. IEEE Software, 22(5), 30-39.

26. Bushuyev S., Yashenko Yu., Tovb A., \& Neizvestny S. (2012, 29-31 October). System paradigm of collective and individual competences of project management specialists. Reports on 26th IPMA World Congress, Greece, Iraklion. 